\title{
Bemerkungen zur Edition
}

Bei der vorliegenden Quellenpublikation handelt es sich um die erste größere Arbeit, die auf marxistisch-leninistischer Grundlage die Niederschlagung des Kapp-Lüttwitz-Putsches durch die einig handelnde deutsche Arbeiterklasse dokumentiert.

Um das Quellenmaterial übersichtlich anzuordnen, haben die Bearbeiter das regional-chronologische Gliederungsprinzip angewandt. Dadurch ist es möglich, das historische Nebeneinander in ein überschaubares Nacheinander zu bringen und die gemeinsamen wie auch unterschiedlichen Formen des Volkskampfes gegen Putschisten und Militaristen in den einzelnen Teilen Deutschlands zutage treten zu lassen. Die Publikation gliedert sich in die folgenden Abschnitte:

I. Vorbereitung des Putsches;

II. Die Niederschlagung des Putsches in den verschiedenen Teilen Deutschlands:

1. Berlin und Umgebung,

2. Land Sachsen,

3. Provinz Sachsen und Anhalt,

4. Thüringen,

5. Niederlausitz, Schlesien, Ostpreußen, Freistaat Danzig,

6. Mecklenburg, Pommern, Wasserkante,

7. Bayern, Baden, Württemberg,

8. Rheinland-Westfalen, Hessen, Saargebiet.

Die Abschnitte „Vorbereitung des Putsches“ und „Berlin und Umgebung" stützen sich vornehmlich auf Archivalien aus dem Zentralen Parteiarchiv des Instituts für Marxismus-Leninismus beim $\mathrm{ZK}$ der SED, den beiden Abteilungen des Deutschen Zentralarchivs in Potsdam und Merseburg, dem Bundesarchiv Koblenz und auf solche überregionale Presseorgane wie die „Rote Fahne“, die „Freiheit" und den 
„Vorwärts“. Für die übrigen Kapitel wurden vorwiegend Materialien der ehemaligen Landeshaupt- und Landesarchive, kommunaler Archive der DDR, Westdeutschlands und der Volksrepublik Polen sowie die regionale Presse herangezogen.

Die Quellen widerspiegeln die Entstehung und Wirksamkeit der Aktionseinheit der Arbeiterklasse, die Durchführung des Generalstreiks, die Zielsetzung der Werktätigen bei Ausbruch und Niederschlagung des Putsches, die Entwicklung bewaffneter Kämpfe der Volkskräfte gegen die konterrevolutionären Truppen sowie die Versuche zur Bildung einer Arbeiterregierung.

Aus den Dokumenten geht hervor, daß unterschiedliche Kampfbedingungen in den einzelnen Teilen Deutschlands bestanden, die abhängig waren von der Stärke der Arbeiterbewegung und von dem Auftreten der örtlichen Konterrevolutionäre. Insgesamt zeigt sich aber, daß ungeachtet regionaler und örtlicher Unterschiede die Arbeiterklasse und alle anderen demokratischen Kräfte das gemeinsame Ziel hatten, durch Schaffung wahrhaft demokratischer Verhältnisse die Wiederkehr von reaktionären Staatsstreichen unmöglich zu machen und daß sich mit der Niederschlagung des Putsches das Kräfteverhältnis zugunsten der Arbeiterklasse verändert hatte. Deutlich wird, daß damit die objektiven Voraussetzungen für die Bildung einer Arbeiterregierung entstanden waren.

Die meisten dieser Quellen werden der Offentlichkeit erstmalig zugänglich gemacht. Einige Dokumente, die von grundlegender Bedeutung für das Verständnis der Politik der Arbeiterorganisationen sind und bereits im Band VII der Reihe „Dokumente und Materialien zur Geschichte der deutschen Arbeiterbewegung" vom Institut für MarxismusLeninismus beim ZK der SED publiziert wurden, erscheinen nochmals in der vorliegenden Arbeit.

Eine wichtige Ergänzung zu den publizierten Quellen stellen die Erinnerungsberichte von Arbeiterveteranen dar, die vor allem anläßlich des 40. Jahrestages der Niederschlagung des Kapp-Putsches erschienen. Sie vermitteln ein lebendiges, auf eigenem Erleben beruhendes anschauliches Bild von den heroischen Kämpfen der Volksmassen im März/ April 1920.

In Ubereinstimmung mit der Dialektik des politischen Kampfes wurden darüber hinaus solche Dokumente aufgenommen, in denen sich die Strategie und Taktik der herrschenden Klasse widerspiegelt. Die auf 
zwei Bände beschränkte Publikation stellt naturgemäß nur eine Auswahl des reichhaltigen Quellenmaterials dar.

Die Quellen werden im allgemeinen vollständig abgedruckt. Eliminierungen wurden nur vorgenommen, wenn es sich um unwesentliche Passagen handelte. Sie werden durch in eckige Klammern gesetzte Punkte oder durch den Vermerk in der Überschrift: „Auszug aus..." gekennzeichnet. Der Inhalt der eliminierten Textstellen ist in Anmerkungen kurz wiedergegeben worden. Außerdem enthält der Anmerkungsapparat politische Einschätzungen und Querverweise auf andere in direktem $\mathrm{Zu}$ sammenhang mit dem vorliegenden Dokument stehende Quellen, Druckvermerke, Erläuterungen für das Verständnis notwendiger Sachverhalte sowie Hinweise auf weiteres nichtveröffentlichtes Quellenmaterial und Literaturangaben. Weiterhin werden in den Anmerkungen wichtige Tatsachen aus Dokumenten angeführt, auf die aus Raumgründen verzichtet werden mußte.

Jede Quelle wird genau gekennzeichnet. Ließen sich fehlende Ortsbzw. Datumsangaben ermitteln, wurden sie in eckigen Klammern gesetzt. Wenn Orts- bzw. Datumsangaben nicht zu eruieren waren, sind die dafür üblichen Abkürzungen verwandt worden. Bei gleichnamigen oder weniger bekannten Presseorganen wird zusätzlich ihr Erscheinungsort angegeben. Zeitungen, die nicht mehr als geschlossener $\mathrm{Be}-$ stand vorhanden und einem Archiv entnommen sind, führen die entsprechende Archivsignatur.

Auf die Angabe der Provenienz folgt die Kennzeichnung des Dokuments nach der systematischen und genetischen Aktenkunde. Sämtliche Unterzeichnungen oder Abzeichnungen werden originalgetreu übernommen. Bei wörtlicher Ubbereinstimmung zwischen Absender und Unterzeichner wird auf die nochmalige Angabe des letzteren verzichtet. Dokumente ohne Unterzeichnung tragen den Vermerk „Ohne Unterschrift“. Rang und Titel von Personen erscheinen entweder in der Uberschrift oder in Verbindung mit dem Namen des Unterzeichners. Bei behördlichen Rundschreiben wird auch der Verteiler angegeben. Im Dokument enthaltene Vermerke, wie „Eilt“, „Geheim“ usw., erscheinen ebenfalls. Handschriftliche Zusätze und Marginalien werden als Anmerkungen mit Kleinbuchstaben am Ende des Dokuments angeführt.

Anfang und Ende des Quellenteiles werden durch französische Anführungszeichen gekennzeichnet. Die Personennamen der Unterzeichner sind gesperrt. Auf Hervorhebungen ist verzichtet worden. Die Texte sind nach der modernen Rechtschreibung und Zeichensetzung bearbeitet. Alle 
Zusätze in eckigen Klammern stammen von den Bearbeitern. Im Schlagwortregister wird die Nummer des betreffenden Dokuments angegeben, während im Personenregister und im Verzeichnis der geographischen Namen die jeweilige Seite angeführt wird. Die vollständigen Archivsignaturen sowie die Gesamttitel der Zeitungen erscheinen in der Übersicht der benutzten Quellen.

Halle/Saale, im November 1969

Erwin Könnemann 